\title{
Specular nonlinear anisotropic polarization effect along fourfold crystal symmetry axes
}

\author{
A. R. Bungay, S. V. Popov, and N. I. Zheludev \\ Department of Physics, University of Southampton, Highfield, Southampton SO17 1BJ, UK \\ Yu. P. Svirko \\ General Physics Institute, 38 Vavilov Street, Moscow 117333, Russia
}

Received September 12, 1994

\begin{abstract}
We present what is to our knowledge the first experimental observation of the specular nonlinear anisotropic polarization effect of a pump-induced polarization-plane rotation for normal-incidence reflection from the (001) surface of a cubic crystal. In GaAs, azimuth rotation of the order of $9 \times 10^{-6} \mathrm{rad}$ is seen for a pump intensity of $75 \mathrm{MW} / \mathrm{cm}^{-2}$ at $750 \mathrm{~nm}$, from which the anisotropic component of the cubic nonlinearity $\mid \operatorname{Re}\left(\chi_{x x x x}-2 \chi_{x x y y}-\right.$ $\left.\chi_{x y y x}\right) \mid=5 \times 10^{-9}$ esu is found.
\end{abstract}

It is sometimes anticipated that the optical properties of cubic crystals are isotropic. This is correct only in a rough approximation and indicates only that the dielectric tensor in cubic point groups is spherically symmetric, $\epsilon_{i j}=\epsilon \delta_{i j}$. Taking into account first-order ${ }^{1}$ and second-order ${ }^{2}$ nonlocal (gradient) terms in the linear optical response ruins spherical isotropy, giving rise to weak birefringence. Even without taking into account nonlocality, anisotropy of cubic crystals is pronounced in nonlinear optics. Deviation from spherical symmetry of the tensor of the third-order nonlinearity leads to the dependence of the efficiency of the two-photon absorption process on crystal orientation. ${ }^{3}$ Nonlinear anisotropy is responsible for one of the basic effects of polarization-sensitive nonlinear optics consisting of intensity-dependent polarization-plane self-rotation of linearly polarized light propagating along a fourfold axis of a cubic crystal. ${ }^{4-7}$ This anisotropy is also responsible for pump-probe polarization effects along fourfold axes. ${ }^{8}$ Cubic anisotropy leads to soft polarization symmetry breaking and to frustrated polarization instability of two counterpropagating waves. ${ }^{9,10}$ The effects of anisotropy of the cubic nonlinearity are not confined to propagation phenomena; they may also appear in reflected light as intensity-dependent polarization alteration. This eventuality was discussed theoretically. ${ }^{11}$ Here we report what to our knowledge is the first observation of pump-probe specular polarization-azimuth rotation resulting from the nonlinear anisotropy of cubic structures and call it the specular nonlinear anisotropic polarization effect (SNAPE).

Let us consider a (001) surface of a cubic crystal stimulated by a linearly polarized pump wave propagating along the [001] direction. The polarization state of a probe wave reflected normally from the same surface will be influenced by the presence of the pump. If the pump and the probe are initially linearly polarized in different directions of the polarization azimuth, the specular optical Kerr effect ${ }^{12}$ would dominate. The specular inverse Faraday effect ${ }^{13-17}$ would be seen if the pump wave is circularly polarized. However, if the pump and the probe are initially identically polarized (see Fig. 1) and effects of nonlocality are ignored, then only SNAPE, i.e., crystal anisotropy, can lead to an alteration of the reflected probe polarization. This anisotropy may be introduced by means of the constitutive equation $D_{i}=\epsilon_{i j} \mathcal{E}+\chi_{i j k l} \mathcal{E}_{j} \mathcal{E}_{k} \mathcal{E}_{l}+\ldots$, where $\mathcal{E}$ is the total electric field of the interacting waves. In cubic crystals the nonlinearity tensor $\chi_{i j l m}(\omega, \omega, \omega,-\omega)$ has three independent components, $\chi_{i i i i}, \chi_{i i j j}$, and $\chi_{i j j i}$. The degree of departure from isotropy may be described by the combination of susceptibilities $\Delta \chi=\chi_{i i i i}-2 \chi_{i i j j}-\chi_{i j j i}$, which is equal to zero in any isotropic medium by symmetry. Phenomenological theory of self-action propagation-type polarization effects owing to this anisotropic combination of components of the cubic nonlinear susceptibility was reported in Refs. 6 and 18, and a similar theory of the self-action reflective effect was introduced in Ref. 11. A wave analysis similar to that in Ref. 17 shows that, in a pump-probe configuration, the alteration of the reflected probe polarization that is induced by the indentically linearly polarized pump, both of which approach the (001) surface along the [001] direction, is also controlled by $\Delta \chi$. The alteration of the polarization state of the reflected probe in SNAPE is given by the following formula:

$$
\begin{aligned}
\left(\begin{array}{c}
\delta \alpha_{r} \\
-\eta_{r}
\end{array}\right)= & \frac{\left|E_{p}\right|^{2}}{2 n\left(1-n^{2}\right)}\left(\begin{array}{c}
\operatorname{Re} \\
\operatorname{Im}
\end{array}\right)\left(\chi_{x x x}-2 \chi_{x x y y}-\chi_{x y y x}\right) \\
& \times\left[1+\frac{1}{2} \exp (i \psi)\right] \sin 4 \Phi
\end{aligned}
$$

Here $E_{p}$ is the electric-field strength of the pump wave, $\epsilon=n^{2}, \psi$ is the relative phase between the incident pump and the probe waves, $\delta \alpha_{r}$ is the change of the polarization azimuth, and $\eta_{r}$ is the degree of ellipticity of the reflected probe wave.

In most practical cases the anticipated magnitude of SNAPE is several orders of magnitude smaller 


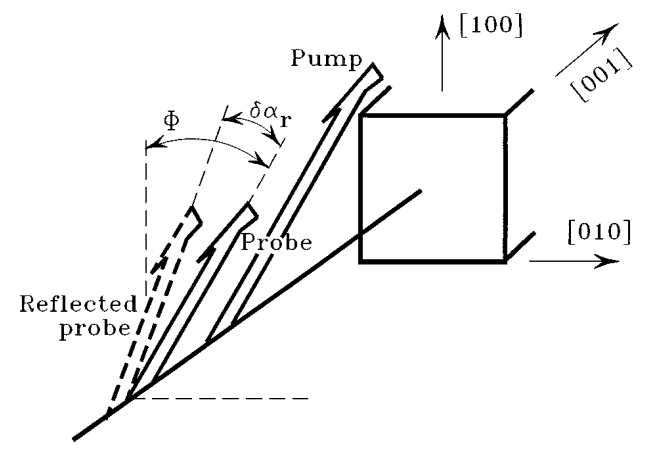

Fig. 1. Observation of SNAPE. The pump and the probe approach the crystal nearly normally to the surface and have identical linear polarizations, making an angle $\Phi$ with respect to the [100] axis of the crystal. The reflected-probe polarization-azimuth rotation is given by $\delta \alpha_{r}$.

than that of the relevant effect in transmission. Equation (1) may be split into two terms. The first one does not depend on the relative phase $\psi$ between the pump and the probe and does not require their mutual coherence to contribute to the SNAPE; this is the incoherent contribution. The last terms containing the exponential factor $\exp (i \psi)$, on the other hand, depend on the relative phase $\psi$ and are responsible for the coherent contribution to SNAPE (compared with the specular inverse Faraday effect $\left.{ }^{14,16,17}\right)$. We measured the incoherent contribution to the rotation of the reflected probe wave polarization azimuth:

$$
\delta \alpha_{r}=\frac{\operatorname{Re}(\Delta \chi)}{2 n\left(1-n^{2}\right)}\left|E_{p}\right|^{2} \sin 4 \Phi .
$$

Our experiments were performed at room temperature with good substrate-quality bulk GaAs crystals cut and polished perpendicular to the [001] direction. We used a polarimeter based on a $\tau_{p}=2 \mathrm{ps}$ Ti:sapphire laser. The polarimeter was set up in the configuration shown in Fig. 1 and had $1-\mu \mathrm{rad}$ sensitivity to polarization-azimuth rotation. In all our experiments the pump and probe waves were initially identically linearly polarized at a wavelength of $750 \mathrm{~nm}$ and focused onto the crystal surface by a lens. At this wavelength, GaAs is completely opaque, with a penetration depth of approximately $\ell=1 \mu \mathrm{m}\left(\hbar \omega \approx 1.65 \mathrm{eV}, E_{g}^{\mathrm{GaAs}} \approx 1.41 \mathrm{eV}\right)$. We studied the reflected probe light polarization-azimuth rotation as a function of the relative pump-probe delay and also measured the dependence of the observed rotation on crystal orientation, i.e., the angle between the incident polarization plane and the [001] axis of the crystal, given by $\Phi$, by rotating the crystal about the [001] direction. The intensity-dependent anisotropic polarization effect reported here was observed on the background of natural polarizationplane rotation, which has a different crystal orientational dependence and which we reported previously. ${ }^{1}$ The polarimetric technique used here and reported in Ref. 19 was sensitive solely to pump-induced polarization-plane rotation of the probe wave while ignoring any natural rotation. It was based on lockin detection with modulated pump intensity and modulated incident-probe polarization azimuth with an amplitude $A$ of approximately $10^{-2} \mathrm{rad}$. If the pumpstimulated reflected-probe polarization azimuth $\delta \alpha_{r}$ changed, then the current of the polarimeter signal channel photodetector had a spectral component at the combined frequency of modulation of the pump intensity and the probe polarization azimuth. The amplitude of this spectral component was proportional to $\delta \alpha_{r} A$, i.e., to $\operatorname{Re}(\Delta \chi)\left|E_{p}\right|^{2} A$ [see Eq. (2)] and was measured by phase-sensitive detection. Correspondingly, the lock-in output voltage was a direct measure of the anisotropic part of nonlinearity. The natural rotation causes photodetector current modulation on the frequency of the probe polarization modulation itself and was totally eliminated by passive filtering and by the lock-in. Also, we could easily check the efficiency of the elimination of the linear signal by blocking the pump beam while setting the polarimeter output to zero.

The time-domain dependence of the rotation of the probe polarization azimuth induced by the pump was examined at several different intensities, and a typical curve is presented in Fig. 2. We point out the sharp peak at the beginning of the curve that coincides with the excitation. We measured the orientational dependence of the observed probe azimuth rotation by rotating the crystal about its [001] axis. The value of polarization-azimuth rotation at the peak, i.e., at zero delay time, has opposite sign for $\Phi \rightarrow-\Phi$ and disappears for incident pump-probe polarization parallel to the [100], [110], or [010] axis of the crystal. In addition, its magnitude oscillates with a dependence of $\sin 4 \Phi$. Consecutive petals have the maximum measured value of $\delta \alpha_{r}=$ $\pm 9 \times 10^{-6} \mathrm{rad}$ for $\Phi=\pi / 8 \pm \pi n / 4$ ( $n$ is an integer) at $I_{\text {pump }}=75 \mathrm{MW} \mathrm{cm}^{-2}$. We attribute the rotation at zero pump-probe time delay to the anisotropy of crystal nonlinearity, as predicted by Eq. (2) (SNAPE). The following value of the anisotropic component of the cubic nonlinearity may be derived from these data: $\left|\operatorname{Re}\left(\chi_{x x x x}-2 \chi_{x x y y}-\chi_{x y y x}\right)\right|=5 \times 10^{-9} \mathrm{esu}$, which is equivalent to $7 \times 10^{-17}$ SI. In this study

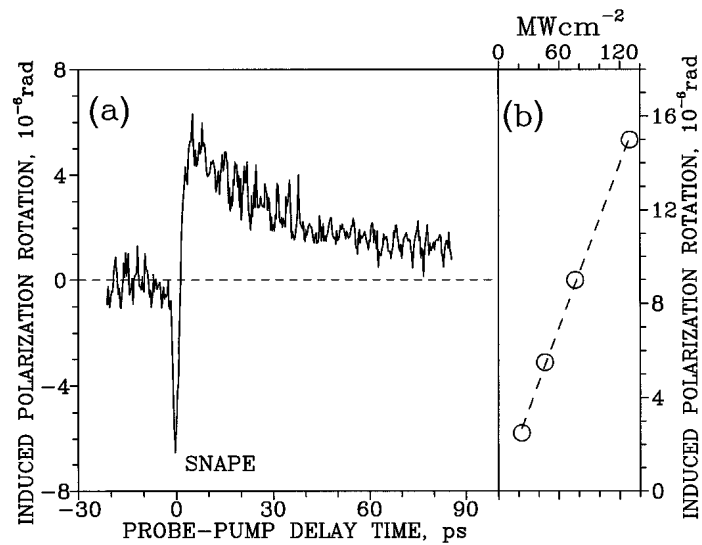

Fig. 2. (a) Induced probe polarization-azimuth rotation as a function of probe-pump delay time for $\Phi=\pi / 8$. The pump intensity is $75 \mathrm{MW} \mathrm{cm}^{-2}$. The sharp peak at the beginning of the curve is due to the anisotropic part of the third-order nonlinearity. (b) Magnitude of the rotation at a probe-pump delay of $-0.5 \mathrm{ps}$ as a function of the pump intensity. 


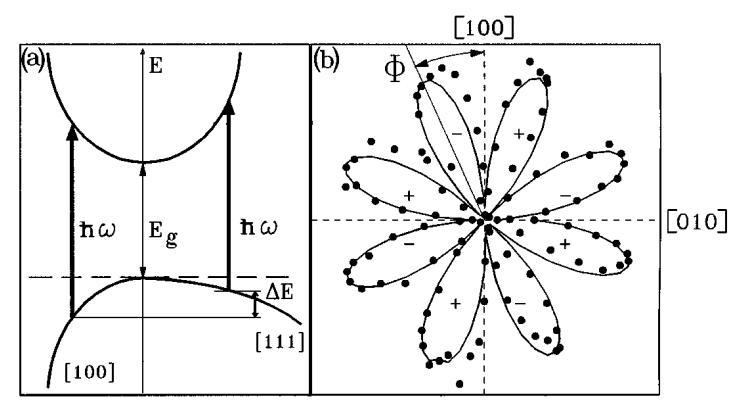

Fig. 3. (a) Fragment of the band structure of GaAs indicates anisotropy of the heavy-hole band, specific for all cubic crystals. (b) Dependence of the observed rotation on $\Phi$ presented in a polar coordinate system, measured at a probe-pump delay of $-0.5 \mathrm{ps}$, meets the $\sin 4 \Phi$ function, as predicted by Eq. (2).

we have particularly concentrated on the results of the observation of this cubic anisotropy. However, the tail of the time-domain dependence (Fig. 2), which we do not discuss here, is due to induced klinear birefringence and was studied previously with a subnanosecond laser. ${ }^{19,20}$

In GaAs the anisotropic nonlinearity $\Delta \chi=\chi_{x x x x}-$ $2 \chi_{x x y y}-\chi_{x y y x}$ may be attributed predominantly to single-photon valence-conductivity band transitions. Taking into account the strong warping of the heavyhole subband is essential for this analysis. This warping reflects the difference in the heavy-hole effective mass for momentum in the [111] and [100] directions: $m_{h h}^{[100]} \approx 0.4 m_{o}$ and $m_{h h}^{[111]}=0.8 m_{o}$ (Fig. 3). For the parameters of the experiment the warping $\Delta_{h h}=m_{c}\left(m_{h h}^{[111]}-m_{h h}^{[100]}\right)\left(m_{c}+\right.$ $\left.m_{h h}^{[111]}\right)^{-1}\left(m_{c}+m_{h h}^{[100]}\right)^{-1}\left(\hbar \omega-E_{g}\right)$ is approximately $17 \mathrm{meV} .{ }^{21}$ The theory of $\Delta \chi$ that accounts for this anisotropy was developed in Refs. 18 and 22, while Ref. 23 gives the classical oscillator theory. In the case of single-photon resonance absorption ${ }^{18}$ the anisotropic nonlinearity has the following dispersion above the main band gap of the crystal: $\operatorname{Im}[\Delta \chi(\omega, \omega, \omega,-\omega)] \propto\left(\hbar \omega-E_{g}\right)^{1 / 2} \cdot{ }^{18}$ The relaxation time of the nonlinearity is controlled by the hole's subpicosecond quasi-momentum relaxation time. This explains why the dynamics of the anisotropic effect are not resolved with our 2-ps laser. This also explains why, with much longer pump and probe pulses of $80 \mathrm{ps}$, the anisotropic effect is completely hidden by the nonlocal polarization effect that has a much longer relaxation time..$^{19,20}$ Although the treatments of Dubenskaya et al. ${ }^{18}$ and Dykman and Rubo ${ }^{22}$ discover the microscopic origin of $\Delta \chi$, their data could not be directly compared with our measurements because the experimentally observed effect is due to $\operatorname{Re}(\Delta \chi)$, whereas they calculate only $\operatorname{Im}(\Delta \chi)$. Concluding, for the first time to our knowledge we have observed a new specular polarization effect owing to the cubic anisotropy (SNAPE). In GaAs it is associated with the band warping.

The authors are grateful to R. Worsley for experimental assistance. This study was partially supported by the R. W. Paul Instrument Fund, the Engineering and Physical Sciences Research Council, UK, and the International Science Foundation (grant N4V000).

\section{References}

1. A. R. Bungay, S. V. Popov, Yu. P. Svirko, and N. I. Zheludev, Chem. Phys. Lett. 217, 249 (1994).

2. P. Y. Yu and M. Cardona, Solid State Commun. 9, 1421 (1971).

3. S. J. Bepko, Phys. Rev. B 12, 669 (1975).

4. M. Ya. Valakh, M. I. Dykman, M. P. Lisitsa, G. Yu. Rudko, and G. G. Tarasov, Solid State Commun. 30, 133 (1979).

5. A. I. Kovrigin, D. V. Yakovlev, B. V. Zhdanov, and N. I. Zheludev, Opt. Commun. 35, 92 (1980).

6. A. D. Petrenko and N. I. Zheludev, Opt. Acta 31, 1177 (1984).

7. R. S. Zadoyan, N. I. Zheludev, and L. B. Meysner, Solid State Commun. 55, 713 (1985); S. A. Akhmanov, B. V. Zhadanov, N. I. Zheludev, N. I. Kovrigin, and V. I. Kuznetsov, JETP Lett. 29, 264 (1979); S. A. Akhmanov, N. I. Zheludev, and R. S. Zadoyan, Sov. Phys. JETP 64, 579 (1987); M. G. Dubenskaya, R. S. Zadoyan, and N. I. Zheludev, J. Opt. Soc. Am. B 2, 1174 (1985).

8. S. P. Apanasevich, D. N. Dovchenko, and N. I. Zheludev, Opt. Spectrosc. (USSR) 62, 481 (1987).

9. J. Yumoto and K. Otsuka, Phys. Rev. Lett. 54, 1806 (1985).

10. For review see N. I. Zheludev, Sov. Phys. Usp. 32, 357 (1989).

11. N. I. Zheludev, A. D. Petrenko, and G. I. Trush, Sov. Phys. Crystallogr. 32, 232 (1987).

12. T. Saiki, K. Takeuchi, M. Kuwata-Gonokami, T. Mitsuyu, and K. Ohkawa, Appl. Phys. Lett. 60, 192 (1992).

13. M. Kuwata, J. Lumin. 38, 247 (1987).

14. S. V. Popov, Yu. P. Svirko, and N. I. Zheludev, Opt. Lett. 19, 13 (1994); A. R. Bungay, A. Malinowski, A. V. Popov, Yu. P. Svirko, R. E. Worsley, and N. I. Zheludev, in International Quantum Electronics Conference, Vol. 9 of 1994 OSA Technical Digest Series (Optical Society of America, Washington, D.C., 1994), pp. $113-114$

15. N. I. Zheludev, M. A. Brummel, R. T. Harley, A. Malinowski, S. V. Popov, D. E. Ashenford, and B. Lunn, Solid State Commun. 89, 823 (1994).

16. N. I. Zheludev, S. V. Popov, A. Malinowski, Yu. P. Svirko, W. Y. Liang, and C. T. Lin, Solid State Commun. 90, 287 (1994).

17. Yu. P. Svirko and N. I. Zheludev, J. Opt. Soc. Am. B 11, 1388 (1994).

18. M. G. Dubenskaya, T. M. Ilyinova, A. V. Trukhov, and A. A. Fortygin, Bull. Russ. Acad. Sci. Phys. Sci. 57, 1192 (1992).

19. N. I. Zheludev, S. V. Popov, A. Malinowski, Yu. P. Svirko, and D. Paraschuk, Phys. Rev. B 50, 11508 (1994).

20. N. I. Zheludev and D. Yu. Paraschuk, JETP Lett. 52 32 (1990).

21. D. N. Mirlin, in Optical Orientation, F. Meier and B. P. Zakharchenia, eds. (North-Holland, Amsterdam, 1984), pp. $141-145$

22. M. I. Dykman and Yu. G. Rubo, Phys. Rev. B 45, 5926 (1992).

23. N. I. Zheludev and Yu. P. Svirko, Sov. Phys. Crystallogr. 35, 153 (1990). 